Original Paper

\title{
Cost Evaluation of In-Space Transportation of a Solar Power Satellite Using OTVs with Hall Thruster Propulsion Systems
}

\author{
By Yuki Ito $^{1)}$, Masakatsu NAKANO ${ }^{2)}$, Tony SCHÖNHERR ${ }^{3)}$, Shinatora $\mathrm{CHO}^{3)}$, Kimiya KOMURASAKI $^{1)}$ and Hiroyuki KoIZUMI ${ }^{4)}$ \\ ${ }^{1)}$ Department of Advanced Energy, The University of Tokyo, Kashiwa, Japan \\ ${ }^{2}$ Department of Engineering, Tokyo Metropolitan College of Industrial Technology, Tokyo, Japan \\ ${ }^{3)}$ Department of Aeronautics and Astronautics, The University of Tokyo, Tokyo, Japan \\ ${ }^{4}$ Research Center for Advanced Science and Technology, The University of Tokyo, Tokyo, Japan
}

(Received June 24th, 2013)

\begin{abstract}
Cost evaluation of a solar power satellite (SPS) in-space transportation using Hall thruster propulsion systems is conducted to obtain specific characteristics of Hall thrusters that yield significant transportation cost reduction. Therefore, the transportation scenario is optimized in the first place: the choice of reusing or disposing orbit transfer vehicles (OTVs), and the power of the propulsion system. The result shows that the case of reusing OTVs is superior to the case of disposing them, because the OTVs' manufacturing costs are predominant in the total cost. In addition, the power has only limited influence on the cost. Further, specific characteristics of Hall thrusters are discussed, which are necessary to achieve a target cost of $\$ 3.75$ billion (300 billion yen) for the sum of two costs: the in-space transportation cost from a low Earth orbit (LEO) to a geostationary Earth orbit (GEO) and the propellant launch cost from Earth to LEO.
\end{abstract}

Key Words: In-Space Transportation, Hall Thruster, Solar Power Satellite, Orbital Transfer Vehicle

\section{Nomenclature}

$c$
$C$
$g$
$i$
$I_{\mathrm{sp}}$
$m$
$M$
$n$
$N$
$p$
$P$
$q$
$r$
$V$
$\Delta V$
$\kappa$

\section{Subscripts}

down

flight

LEO-GEO

prop.

prop-launch

total

up

vehicle cost of one module / one flight

integrated cost of entire mission

gravitational constant

angle of the orbit inclination of $30.4^{\circ}$

specific impulse

mass of one module / one flight

integrated mass of entire mission

number as variable

integrated number of entire mission

power of single thruster system

propulsion power of OTV

thruster cost coefficient

learning rate of 0.85

orbital velocity

velocity increment

power contribution factor from SPS

transportation from GEO to LEO

round trip between LEO and GEO

transportation between LEO and GEO

propellant

propellant launch from Earth to LEO

total

transportation from LEO to GEO

OTV

\section{Introduction}

High-power electric propulsion systems ranging from few to hundreds of $\mathrm{kW}$ are actively studied ${ }^{1,2)}$ in recent years. Electric propulsion systems of this power range can be applied to main thrusters on spacecrafts causing the propellant consumption to be reduced by one order of magnitude. As a result, the space mission capability is enhanced. Satellites recently started to employ $5 \mathrm{~kW}$ electric propulsion systems ${ }^{3)}$, and electric propulsion systems with high power would improve the feasibility of space programs relying on in-space transportation of huge payload such as SPS especially regarding transportation costs. For those high-power electric propulsion systems, Hall thrusters are considered to be most suitable among the various kinds of electric propulsion systems ${ }^{4}$. Therefore, this study focuses on the derivation of specific characteristics of Hall thrusters that will yield significant cost reduction of in-space transportation of a SPS.

The concept of SPS is to generate power in orbit and transmit the power to Earth ${ }^{5)}$. SPSs have several advantages: 1) SPSs utilize renewable energy, 2) photovoltaic cells yield 5 - 10 times higher power in orbit compared to the ones on Earth $^{6}$, 3) power generation of SPS is stable. Among these advantages, the stability of power generation is most important, as the abrupt fluctuation of power generation by renewable energy is a continuing issue in this field ${ }^{6)}$. However, the typical mass of a SPS is in the range of tens of thousands of tons making it difficult to construct a SPS system using current transportation means.

This paper shows parametric studies of the cost evaluations of in-space transportation of a SPS using Hall thrusters. At first, Section 2 shows the transportation scenario which is the 
base scenario of the following parametric study, and Section 3 describes the method of cost evaluation. Then, the optimized mission scenario is discussed from the viewpoint of transportation cost in Section 4, and, finally, the specific characteristics are proposed in Section 5. Section 5 also describes the desirable technologies that enhance the feasibility of in-space transportation of a SPS.

\section{Base Transportation Scenario}

SPS will be operated in a GEO, a circular orbit $36,000 \mathrm{~km}$ above the Earth's equator as exemplarily shown in Figure 1. SPS transportation can be divided into two parts as shown in Figure 2, the launch from Earth to a LEO, a circular orbit 400 $\mathrm{km}$ above ground, and the in-space transportation from LEO to GEO, which is the scope of this study. The usage of OTVs in the in-space transportation was assumed in this study, and the merit of employing OTVs will be discussed in Section 4.1. The OTV was modeled by two major elements; a thruster system and structures. The thruster system includes entire elements which is necessary to operate electric thrusters: thruster head, gimbal, PPU, cabling, propellant feed system, and tank.

To construct a SPS, huge massive structures of several 1,000 to 10,000 tons need to be transported into GEO. This great amount of payload inevitably leads to the employment of separated transfers in individual modules. The SPS mission assumptions used in this study are summarized in Table 1. In this study, the transportation cost from LEO to GEO was evaluated to provide a framework of SPS transportation from the viewpoint of costs. In addition, the cost to launch the propellant consumed in this in-space transportation was also evaluated, because the amount of propellant changes depending on the in-space scenario. The target cost for this transportation was assumed to be $\$ 3.75$ billion (300 million yen), because the entire transportation of SPS has to be accomplished within the expense of $\$ 12.5$ billion (1 trillion yen) to make it competitive enough compared with other power sources. For the launch vehicle used from the Earth to LEO, H-IIB was assumed. Thus, our evaluation started from the 19 tons of mass supply at LEO, which is the maximum payload of $\mathrm{H}-\mathrm{IIB}^{7)}$.

Although there are many candidates for the electric propulsion (EP) system, recent researches ${ }^{4}$ show that Hall thruster would be the most promising choice for SPS orbit transfer vehicles (OTVs). Hall thruster has high thrust efficiency, and the specific power, the propulsion power per unit mass, of Hall thruster is also high. Although the desirable power of SEP exceeds $100 \mathrm{~kW}$ as will be shown in Section 4.2, clustering of $25 \mathrm{~kW}$ thrusters was presumed in this study, because cluster systems would be employed in space programs as a concrete measure against failures.

As propellant for Hall thruster, xenon is usually selected as the ideal candidate to obtain an optimum thrust performance. Xenon is, however, considerably expensive and rare as shown in Table 2, and is not a preferable choice for the mass transportation which consumes huge amount of propellant. Therefore, argon was selected as alternative propellant in this study, and, thus, specific impulse was assumed to be 4,000 s. Although the thrust efficiency tends to be lower if argon is employed, the two order of magnitude cost cut compared with xenon can be expected to raise the feasibility of this mission. Taking the use of argon into account, the thrust efficiency was assumed to be $50 \%$ at first, and was varied later on as a parameter in order to see the impact of the efficiency on the transportation costs.

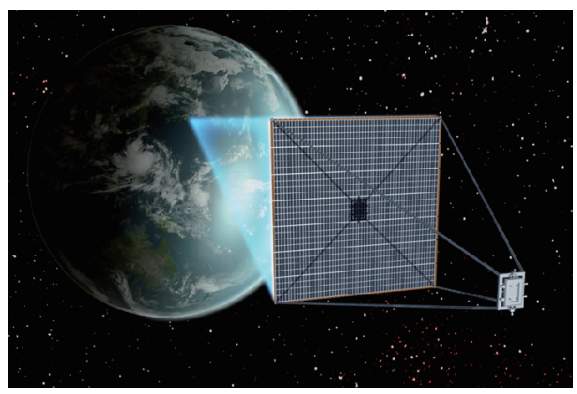

Fig. 1. SPS planned by USEF ${ }^{6)}$.

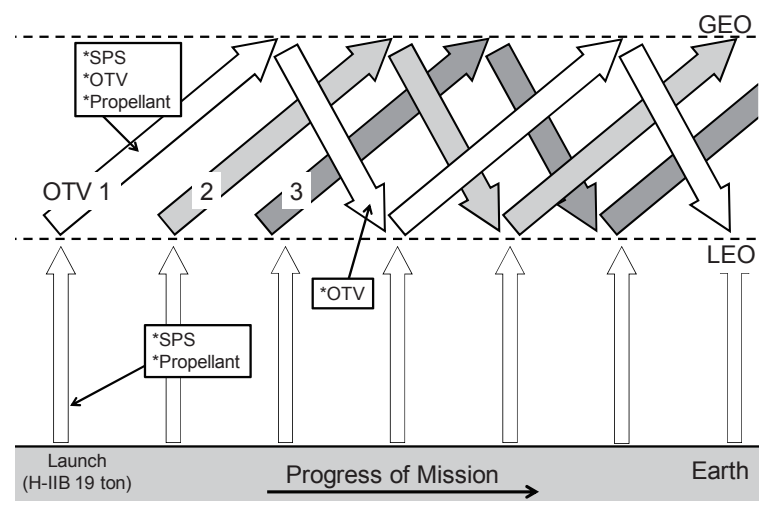

Fig. 2. SPS transportation scenario, where the number of OTV $N_{\text {vehicle }}$ is set to three.

Table 1. Requirements of SPS

\begin{tabular}{|l|l|}
\hline Weight & 20,000 tons \\
\hline Power output & $5 \mathrm{GW}$ \\
\hline \multirow{3}{*}{ Cost target } & $\begin{array}{l}\text { Total: \$12.5B } \\
\text { LEO to GEO: \$3.75B } \\
\text { (Including propellant launch cost } \\
\text { from Earth to LEO) }\end{array}$ \\
\hline Mission period & 10 years \\
\hline
\end{tabular}

Table 2. Properties of propellant.

\begin{tabular}{|l|l|l|l|}
\hline & $\mathrm{Xe}$ & $\mathrm{Kr}$ & $\mathrm{Ar}$ \\
\hline Price $\$ \mathrm{~kg}$ & 1,200 & 330 & 5 \\
Specific impulse s & 2000 & 3000 & 4000 \\
\hline
\end{tabular}

\section{Evaluating Method}

To deduce the optimal transportation scenario, several mission cases were evaluated. The total transportation cost is estimated by:

$$
C_{\text {total }}=C_{\mathrm{LEO}-\mathrm{GEO}}+C_{\text {prop-launch }} .
$$

The elements of transportation from LEO to GEO can also be divided into two parts from the viewpoint of costs: propellant and OTVs. Thus, in-space transportation cost is estimated by 
Table 3. Breakdown of OTV setup and parameters.

\begin{tabular}{|c|c|c|}
\hline & Mass & Cost \\
\hline Propellant (argon) & calculated by Eq.(5) & $\$ 5$ per $1 \mathrm{~kg}$ \\
\hline Thruster system & - & \multirow{5}{*}{$\$ 15$ million per $25 \mathrm{~kW}$} \\
\hline Heads, gimbals, propellant feed system & $1.8 \mathrm{~kg} / \mathrm{kW}^{4)}$ & \\
\hline PPUs & $1.7 \mathrm{~kg} / \mathrm{kW}^{4}$ & \\
\hline Tank & $4 \%$ of propellant mass & \\
\hline Solar array on OTV & $6.0 \mathrm{~kg} / \mathrm{kW}$ & \\
\hline Structure & $10 \%$ of sum of above & $\$ 250$ thousand per 1 ton \\
\hline
\end{tabular}

the following equation:

$$
C_{\text {LEO-GEO }}=N_{\text {flight }} \cdot c_{\text {prop. }}+N_{\text {vehicle }} \cdot c_{\text {vehicle }}^{*},
$$

where $c_{\text {prop }}$ is the cost of propellant per unit mass, $N_{\text {vehicle }}$ is the number of OTVs necessary to complete transportation within the given mission period. The superscript * indicates the consideration of cost saving along the experience curve. The experience curve is representing the increasing efficiency through the repetitive production. The experience curve is usually expressed by Eq. $(3)^{8}$, showing a relationship between the production cost and the cumulative production number.

$$
c_{\text {vehicle }}(n)=r^{\log _{2}(n)} c_{\text {vehicle }}(0) \text {. }
$$

As Eq. (3) shows, when the cumulative production number is doubled, the production cost is multiplied by $r$. In this paper, $r$ is assumed to be 0.85 with reference to aircraft manufacturing ${ }^{9)}$. Thus, the integrated manufacturing cost of OTV is calculated using Eq. (4). The impact of repetitive production on cost derived from Eq. (4) is shown in Figure 3.

$$
N_{\text {vehicle }} \cdot c_{\text {vehicle }}^{*}=\sum_{n=1}^{N_{\text {velicle }}} r^{\log _{2}(n)} c_{\text {vehicle }}(0) \text {. }
$$

The propellant cost is proportional to its mass which is obtained by:

$$
m_{\text {prop }}=m_{0} \cdot\left\{1-\exp \left(-\frac{\Delta V}{g I_{\text {sp }}}\right)\right\},
$$

where $m_{0}$ is the initial mass of spacecraft. $\Delta V$ is calculated from the Edelbaum's equation:

$$
\Delta V=\sqrt{V_{\mathrm{LEO}}^{2}+V_{\mathrm{GEO}}^{2}-2 V_{\mathrm{LEO}} V_{\mathrm{GEO}} \cos \left(\frac{\pi}{2} \Delta i\right)},
$$

where $i$ is set to $30.4^{\circ}$ assuming the launch from Tanegashima, Japan. The calculated velocity increment is $\Delta V=6.1 \mathrm{~km} / \mathrm{s}$. Using Eq. (5) and the $\Delta V$, the mass of propellant necessary for OTV transferring from LEO to GEO was acquired by setting $m_{0}$ to the sum of H-IIB payload mass (=19 ton) and OTV dry mass. The propellant mass for the return trip from GEO to LEO is calculated based on the final mass of this flight: OTV mass without propellant.

The OTV cost $c_{\text {vehicle }}$ is calculated using two models: one simulates the thruster system cost and the other simulates the structure cost. The thruster system includes thruster heads, gimbals, propellant feed systems, PPUs, a propellant tank and solar array. The structure is a framework to integrate these elements into a single system. The detailed configuration is given in Table 3. The cost of thruster systems, which include thruster head, gimbal, PPU, propellant feed system, and tank, is determined by a model ${ }^{10)}$ obtained by extrapolating the current market prices. The model assumes that the cost of thruster systems would be the function of output power of thruster system as expressed in Eq. (7).

$$
c_{\text {thruster }}(p)=q \log \frac{p}{p_{0}} \times c_{\text {thruster }}\left(p_{0}\right) .
$$

In this model, the constants are set as follows; $p_{0}=5 \mathrm{~kW}$ and $c_{\text {thruster }}\left(p_{0}\right)=\$ 8$ million, and $q=3$. The factor $q$ was 2 in the reference ${ }^{10)}$, though, 3 is employed taking into account the inaccuracy of extrapolating.

The number of round trip between LEO and GEO $N_{\text {flight }}$ can be obtained by dividing the total SPS mass by the single modularized SPS mass: the H-IIB payload mass (=19 ton) subtracted by the propellant mass $m_{\text {prop }}$ and associated tank mass.

The number of OTV $N_{\text {vehicle }}$ is determined from Eq. (8) using $N_{\text {flight }}$, transfer orbit duration (TOD) of each flight and entire mission period. This equation represents the scenario when OTVs are utilized most efficiently:

$$
N_{\text {vehicle }}=\frac{1}{\frac{\text { MissionPeriod }}{\text { TOD }}} \cdot N_{\text {flight }} .
$$

The cost for propellant launch was calculated assuming that the target cost of SPS launch from Earth to GEO of $\$ 8.75$

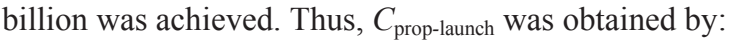

$$
C_{\text {prop-launch }}=\frac{C_{\mathrm{SPS}-\text { launch }}}{M_{\mathrm{SPS}}} \cdot M_{\text {prop }} \text {, }
$$

where the target cost of SPS launch $C_{\text {SPS-launch }}$ is $\$ 8.75$ billion, $M_{\mathrm{SPS}}$ is 20,000 ton, and $M_{\text {prop }}$ is the mass of propellant consumed through the entire in-space transportation.

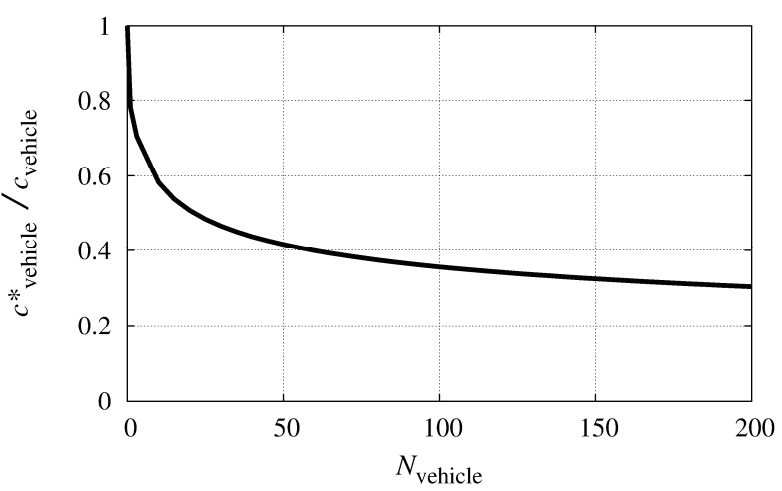

Fig. 3. Impact of repetitive production on cost assumed in this study, where cost of one OTV $c^{*}$ vehicle is normalized by the cost without considering experience curve $c_{\text {vehicle }}$ 
Table 4. Cost comparison of two OTV scenarios.

\begin{tabular}{|l|l|l|l|l|}
\hline$c_{\text {thruster }}$ & Scenario & LEO-GEO & $\begin{array}{l}\text { Propellant } \\
\text { launch }\end{array}$ & Total \\
\hline \multirow{2}{*}{ \$15 million } & reusing & \$3.84 B & \$1.63 B & \$5.47 B \\
& disposing & \$19.7 B & \$1.52 B & $\mathbf{\$ 2 1 . 2 ~ B}$ \\
\hline \multirow{2}{*}{ \$7.5 million } & reusing & \$2.09 B & \$1.63 B & $\mathbf{\$ 3 . 7 2 ~ B}$ \\
& disposing & \$10.6 B & \$1.52 B & $\mathbf{\$ 1 2 . 1 ~ B}$ \\
\hline
\end{tabular}

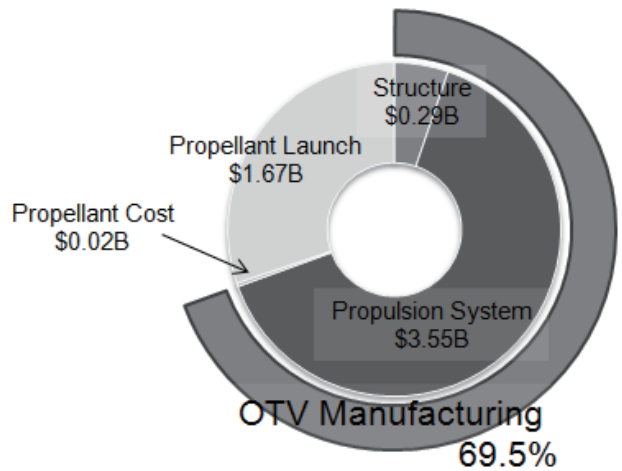

Fig. 4. Cost breakdown of the reusing scenario where $c_{\text {thruster }}=\$ 15$ million.

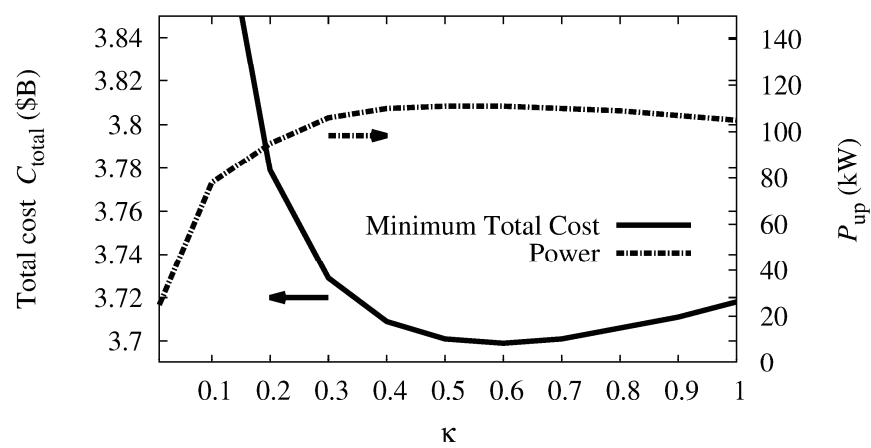

Fig. 5. Relationship between $\kappa$, defined by Eq. (10), and the minimum total cost (left ordinates), and the associated $P_{\text {up }}$ (right ordinates).

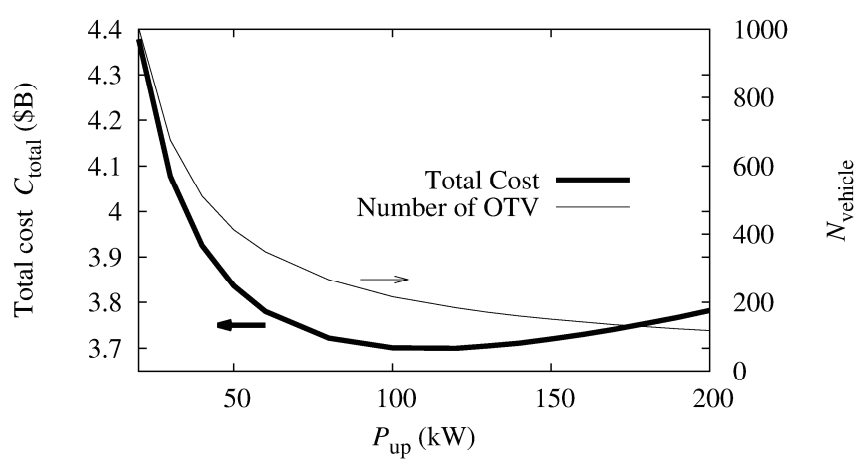

Fig. 6. Relationship between $P_{\text {up }}$ and the total cost (left), and $N_{\text {vehicle }}$ (right), where $\kappa$ was set to 0.6 considering the result of Section 4.2.

\section{Optimum Scenarios}

This section shows the optimum scenarios deduced following the method described in the previous section. Two factors are considered here: the choice of reusing or disposing the OTVs, and propulsion power.

\subsection{OTV scenario}

A comparative study is conducted to derive which option is better: to restrain the number of OTVs by having them to LEO after unloading the payload at GEO and reuse them; or to avoid the flight back to LEO. In this section, propulsion power is assumed to be $125 \mathrm{~kW}$.

The upper row of Table 4 shows the calculation result, where $c_{\text {thruster }}=\$ 15$ million is the cost of the thruster system derived from Eq. (7). The better choice is to reuse OTVs rather than disposing them, as the reusing scenario yields a $74 \%$ lower cost of $\$ 5.47$ billion.

However, the cost of reusing scenario is still higher than the target cost of $\$ 3.75$ billion. In this case, the expense of OTVs' manufacturing cost is as huge as almost $70 \%$ of the total cost as shown in Figure 4. Therefore, another cost estimation was conducted assuming the cost of the propulsion system to be low enough so that the total cost could be kept below the target $\$ 3.75$ billion. The target cost was achieved as shown in lower row of Table 4 when the cost of a $25 \mathrm{~kW}$ Hall thruster is reduced to as low as $\$ 7.5$ million, half of the value used in the previous estimation. The desirable improvements required for the realization of such a cost-down will be discussed in Section 5.

\subsection{Propulsion power and power contribution from SPS}

The propulsion power of OTV is another important factor for the cost, because it affects the number of thruster systems and leads to the changes of TOD. This power can be provided by both the OTV's solar array and the SPS module during the transportation from LEO to GEO, whereas the power from the SPS module will no longer be achievable from GEO to LEO. As the mass of solar array is relatively high, the power contribution from the SPS module would reduce the considerable mass and cost. In this section, the optimum propulsion power during the transportation from LEO to GEO $P_{\text {up }}$ and the optimum power from GEO to LEO $P_{\text {down }}$ which is described using $\kappa$,

$$
P_{\text {down }}=\kappa P_{\text {up }} .
$$

Figure 5 shows the minimum cost as a function of $\kappa$. The minimum point was obtained by evaluating the cost for the wide range of power at each $\kappa$. In addition, the power at which the cost yielded a minimum value is also plotted in Figure 5 using right ordinate. Small value of $\kappa$ denotes the expanded use of SPS as the power source to drive OTVs. The remarkable cost increase in the low $\kappa$ range can be explained by the rapid increase of TOD of return journey. This is because the mass of thruster system for $P_{\text {up }}-P_{\text {down }}=(1-\kappa) P_{\text {up }}$ will perform only as dead mass in the transportation from GEO to LEO. It is concluded that the utilization of SPS as power source has little merit.

The relationship between the power and the cost is described in Figure 6, where $\kappa$ was set to 0.6 considering the previous result. $P=112 \mathrm{~kW}$ yielded the minimum cost where the effect of decreasing TOD and growing OTV manufacturing cost were balanced. From Figure 5 and Figure 6 , it is clear that as long as the power and $\kappa$ was set around the favorable value, the cost is not very sensitive to these parameters. However, a power of less than $100 \mathrm{~kW}$ should be 
avoided, because TOD increases drastically with the decrease of the power as described in Figure 6. This is important, because a radiation belt exists between LEO and GEO which OTVs would inevitably pass through, and the longer TOD leads to a greater exposure to space radiation hazardous to the SPSs' electric circuits. However, equipment which is strong against space radiation is being developed rapidly ${ }^{11}$. Thus, in order to focus on technologies of Hall thrusters, the effect of space radiation is not considered in this study.

\section{Specific Characteristics}

This section describes the specific characteristics of Hall thruster, which leads to an achievement of target transportation cost of a SPS.

\subsection{Propellant}

In this study, argon has been considered as propellant, because the price of argon is quite low as shown in Table 2 . However, the current research on use of argon is limited, and the efficiency of Hall thruster with argon still remains unclear. Thus, this section describes the derivation of the influence of both the choice of propellant and the thrust efficiency on the total cost. Three propellants are considered: xenon, krypton, and argon. In this calculation, specific impulses are assumed as shown in Table 2.

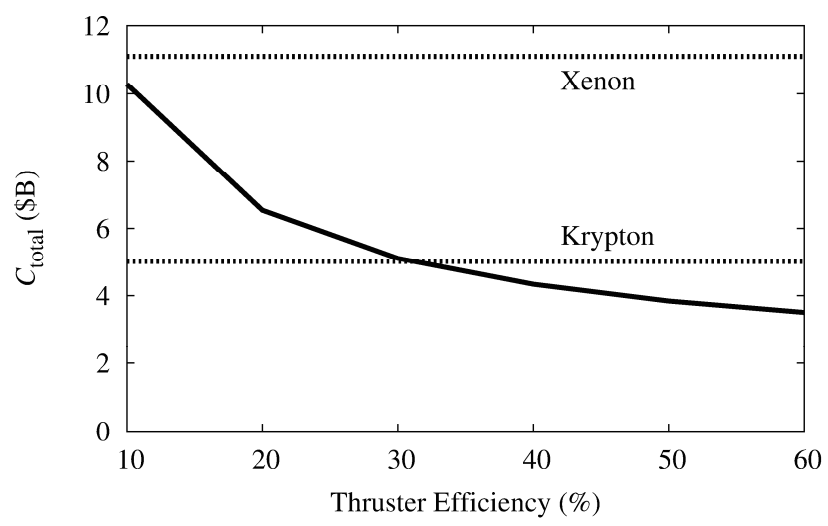

Fig. 7. Relationship between thrust efficiency and total cost with propellant of argon (Thrust efficiency, Kr: 50\%, Xe: 60\%).

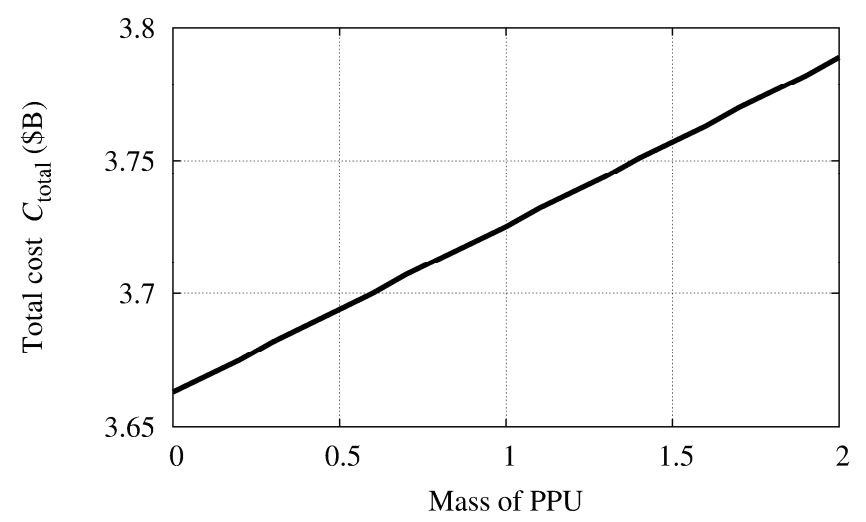

Fig. 8. Relationship between mass of PPU and total cost, where mass of PPU was normalized using previously assumed value of $1.7 \mathrm{~kg} / \mathrm{kW}$.
The result is shown in Figure 7. Selecting argon as propellant provides significant cost reduction at a high thrust efficiency range. However, the cost for the case of a thrust efficiency of below $30 \%$ with argon exceeds that with krypton. Thus, developing a thruster with a thrust efficiency of more than $30 \%$ with argon is desired.

\subsection{Thrust efficiency}

The impact of thrust efficiency was derived as shown in Figure 7. In the case of argon, the result shows that $10 \%$ growth in thrust efficiency offers about $\$ 400$ million cost reduction around efficiency of $50 \%$. As the target cost is $\$ 3.75$ billion, efforts to improve this efficiency should be conducted to enhance the mission feasibility.

\subsection{Thruster systems manufacturing cost}

The introduced cost evaluation gives rise to a demand for low-cost thruster systems. Thus, this section shows two researches that have a potential to reduce transportation costs.

One technology is the channel erosion restriction of Hall thrusters leading to longer lifetime. The lifetime of Hall thrusters have significant influence on the transportation cost, because the manufacturing cost of thruster systems are high. In this study, the lifetime is assumed to be 10 years considering future innovations related to lifetime. However, if the lifetime were 5 years, the transportation cost would increase by $45 \%$. Therefore, the technologies to increase the lifetime are quite important. The lifetime of Hall thrusters is currently determined by the rate of channel erosion. A recent study shows the prospects of Hall thrusters to restrain the channel erosion significantly ${ }^{12)}$, thus, studies to establish the erosion restriction technologies are aimed for.

The other technology is the development of TAL. There are two types of Hall thruster, magnetic layer type (SPT) and anode layer type (TAL). As the market is currently dominated by SPT, the cost model assumed in this study is also based on that of the SPT's. TAL performs, however, with a higher thrust density compared with SPT, and has the potential of lower manufacturing $\cos \mathrm{t}^{13)}$. In addition, the fact that TAL use smaller amount of insulator, which is a costly material because of its poor formability, will also support the prospects of cost reduction. Also, the efficiency of TAL is, in principle, higher than that of SPT's, which would reduce the transportation cost. On the other hand, the shortage of TAL is its discharge oscillation and operation instability, which are undesirable aspects for satellites. Therefore, the development of stable and low-oscillation TAL is envisaged. Studies to reduce oscillation are frequently conducted. One is to feed propellant in a novel way, which was shown to have a good effect on the oscillation restraint ${ }^{14)}$. The other is to provide a smart PPU controlling its power supply to reduce the oscillation $^{15)}$. Such PPUs, however, tend to be heavier than the current PPUs. Thus, the relationship between mass of PPU and the cost is deduced as shown in Figure 8. The result shows that $10 \%$ increment of mass of PPU imposes only $0.3 \%$ increment of the total cost. As the impact of mass of PPU on the total cost is, thus, quite small compared to thrust efficiency, this PPU can be expected to realize stable and low-oscillation TAL. 
Table 5. Optimum scenario of SPS transportation (The cost of thruster systems $(25 \mathrm{~kW})$ was assumed to be one of fourth of that of the state of the art.).

\begin{tabular}{|l|l|l|}
\hline Parameters & Optimum value & Impact on cost \\
\hline OTV scenario & Reuse OTV & $\begin{array}{l}\text { if disposed, } \\
\text { cost is 4 times higher }\end{array}$ \\
\hline Propellant & Argon & $\begin{array}{l}\text { if xenon, } \\
\text { cost is 3 times higher }\end{array}$ \\
\hline Thrust efficiency & $\begin{array}{l}\text { the higher } \\
\text { the better }\end{array}$ & $\begin{array}{l}10 \% \text { eff. improvement, } \\
10 \% \text { cost reduction }\end{array}$ \\
\hline Thrust power & $112 \mathrm{~kW}$ & small, Fig. 7 \\
\hline Power contribution from SPS & $40 \%$ from SPS & small, Fig. 6 \\
\hline
\end{tabular}

\section{Conclusions}

In-space transportation of SPS was examined and the following knowledge was obtained, which is also shown in Table 5. A better choice of OTV scenario was reusing OTVs rather than disposing them, because the manufacturing cost of OTVs was the dominant part of the expenses, occupying $70 \%$ of the total cost. In addition, the power of OTV has limited impact on the total cost, and therefore OTVs can choose its power from a wide range. The fraction of power dependence on SPS during the flight from LEO to GEO yielded no advantage from the viewpoint of cost as described in Section 4.2 .

The cost of the optimal scenario reflecting this knowledge is shown in Figure 4. Based on the results, the specific characteristics of Hall thrusters suitable for in-space transportation of a SPS are presented as follows:

- Capability of operation with argon

- Improved thrust efficiency

- Long lifetime

- Restrained discharge oscillation in case of TAL

\section{References}

1) Arastu, A.: Advances in Technologies for High Power SEP Missions, 10th International Energy Conversion Engineering Conference, Atlanta, Aug. 2012, AIAA 2012-3977.

2) Schönherr, T., Cho, S., Koizumi, H., Komurasaki, K., Yamamoto, N., Miyasaka, T., Kuninaka, H.: Japan's Itinerary Towards
High-Power Electric Propulsion, 4th Russian-German Conference on Electric Propulsion, Moscow, Dec. 2012, No. 60.

3) Gollor, M., Boss, M., Cruz, F., Galantini, P., Bourguignon E. Electric Propulsion Electronics Activities in Europe - 2011, 47th Joint Propulsion Conference, San Diego, Aug. 2011, AIAA 2011-5517.

4) Brophy, R., Gershman, R., Strange, N., Landau, D.: 300-kW Solar Electric Propulsion System Configuration for Human Exploration of Near-Earth Asteroids, 47th Joint Propulsion Conference, San Diego, Aug. 2011, AIAA 2011-5514.

5) Peter G.: Method and Apparatus for Converting Solar Radiation to Electrical Power, U.S. Patent and Trademark Office, 1973.

6) Sasaki, S., Tanaka, K., Okuizumi, N., Kawasaki, S., Shinohara, M., Senda, K., Ishimura, K. and USEF SSPS Study Team: Tethered Solar Power Satellite, JAXA Research and Development Report, JAXA RR-03-005E, (2004), pp. 1-12.

7) Sasaki, H., Imada, T., Takata, S.: Development Plan for Future Mission from HTV System, Transaction of the Japan Society for Aeronautical and Space Sciences, 7 (2010), pp. Tk 77 - 82.

8) Yelle, L.: The Learning Curve: Historical Review and Comprehensive Survey, Decision Sciences, 10 (1979), pp. 302-328.

9) Alchian, A.: Reliability of Progress Curves in Airframe Production, Econometrica, 31 (1963), pp. 679-693.

10) Hofer, R., Randolph, T.: Mass and Cost Model for Selecting Thruster Size in Electric Propulsion Systems, 47th Joint Propulsion Conference, San Diego, Aug. 2011, AIAA 2011-5518.

11) Bailey, S., McNatt, J., Raffaelle, R., Hubbard, S., Forbes, D., Fritzenmeier L., Maurer W.: The Future of Space Photovoltaics, Photovoltaic Specialists Conference, June 2009, pp. 001909 001913.

12) Mikellides, I., Katz, I., Hofer, R., Goebel, D.: Design of a Laboratory Hall Thruster with Magnetically Shielded Channel Walls, Phase III Comparison of Theory with Experiment, 48th Joint Propulsion Conference, Atlanta, Aug. 2012, AIAA 2012-3789.

13) Schönherr, T., Hosoda, M., Cho, S., Koizumi, H., Arakawa, Y., Komurasaki, K., Yamamoto, N.: Low-cost $20 \mathrm{~kW}$ Hall Thruster for Mass Transportation, Asian Joint Conference on Propulsion and Power, Xi'an, Mar. 2012, AJCPP2012-022.

14) Fukushima, Y., Yokota, S., Komurasaki, K., Arakawa, Y.: Influence of Azimuthally Nonuniform Propellant Flow Rate on Thrust Vector and Discharge Current Oscillation in a Hall Thruster, Transaction of the Japan Society for Aeronautical and Space Sciences, 7 (2009), pp. Pb 41 - 45.

15) Tamida, T., Nakagawa, T., Suga, I., Osuga, H., Ozaki, T., Matsui, K.: Determining parameter sets for low-frequency oscillation-free operation of Hall thruster, Journal of Applied Physics, 102 (2007), pp. 043304. 